\title{
Spin: modificações na redação científica que escondem fragilidades metodológicas com impacto social negativo
}

Charles Phillipe de Lucena Alves. Departamento de Educação Física, Centro de Ciências da Saúde, Universidade Federal do Rio Grande do Norte. Rio Grande do Norte, Natal, Brazil. charleslucena@ufrn.edu.br (ORCID 0000-0001-76980621)

Gabriel Gonçalves da Costa. Instituto de Bioquímica Médica Leopoldo de Meis, Universidade Federal do Rio de Janeiro. Rio de Janeiro, Brazil. gabriel.costa@biogmed.ufri.br (ORCID 0000-0002-1141-7827)

João de Deus Barreto Segundo. Escola Bahiana de Medicina e Saúde Pública. Salvador, Bahia, Brazil. nucc-joaosegundo@bahiana.edu.br (ORCID 0000-00024802-6045)

Lucas Helal. Universidade do Extremo Sul Catarinense. Criciúma, Santa Catarina, Brazil, 88806-000 Ih@unesc.net (ORCID 0000-0002-6900-7185)

\section{Autor de correspondência}

Autor de correspondência

Prof. Lucas Helal

Universidade do Extremo Sul Catarinense (UNESC)

Av. Universitária, 1105, Universitário, Criciúma, Santa Catarina, Brasil, 88806000

+55 48 3359-2572

lh@unesc.net

\section{Contribuição dos autores}

$C L$ foi responsável pela concepção da ideia original. Todos os autores, nesse caso, CL, GC, JDB e LH participaram de todas as etapas, a saber: concepção do artigo, escrita, revisão e aprovação final.

\section{Potenciais conflitos de interesse}

Os autores não possuem conflitos de interesse a declarar. 


\section{INTRODUÇÃO}

\section{O que é spin?}

"Uma cura para a calvície pode estar a caminho". A frase chama a atenção e gera visualizações quando afirmada no título ou texto em algum artigo (estudo científico) ou notícia jornalística, correto? O problema é que ela pode dizer respeito a um resultado de um estudo científico que não foi realizado em humanos (ou um estudo que sequer avaliou a calvície propriamente). Sabe-se que modelos animais podem não indicar causalidade (estabelecimento de relações de causa e efeito - nota: ver tabela 1 para definição) para humanos, sendo o estabelecimento da causalidade um dos objetos finais da pesquisa biomédica e para o qual almeja-se o desenvolvimento de inovações em prol da melhoria da saúde e da qualidade de vida da população em geral.

Em termos gerais, qualquer ato (intencional ou não intencional), que deturpa os resultados do estudo no intuito de destacar/enfatizar o efeito benéfico de uma determinada intervenção/procedimento (ou mesmo implicar um efeito benéfico quando este não existe), mesmo na ausência de dados para apoiar as conclusões, pode ser chamado de spin (1). Neste sentido, é um termo utilizado em grande parte (mas não somente) para estudos de característica clínica e/ou intervencionista (de natureza experimental, por exemplo), como estudos que ofertam uma droga ou outra intervenção (A) para um determinado grupo de pacientes ou animais, e uma droga ou outra intervenção (B) ou placebo para outro grupo de pacientes ou animais e que ao final querem saber se a droga $A$ é melhor (ou equivalente) que a droga $B(2,3)$.

Neste sentido, tem-se que um artigo pode mostrar em seus resultados a ineficácia de um determinado procedimento em relação a um placebo (substância ou tratamento inócuo) ou a um tratamento rotineiro, no entanto, a redação do artigo pode se inclinar para convencer os leitores de que o procedimento experimental relatado é superior ou causa benefícios, caracterizando um tipo de spin já documentado(4). As estatísticas podem mentir se manipuladas de determinadas formas, auxiliando a compor uma narrativa irreal dos resultados (5).

Na pesquisa biomédica, o termo spin recebe outros nomes/sinônimos (6), como: apresentação distorcida $(1,7)$, deturpação $(3,8)$, exagero nos resultados da pesquisa (9-11), relatórios enganosos ou inadequados $(12,13)$, interpretação 
tendenciosa (14), superinterpretação (7) ou interpretação incorreta e extrapolação inadequada (8).

Neste sentido, a presença de spin pode dificultar ou distorcer a tradução da pesquisa para a prática clínica, fornecendo expectativas irreais sobre novos tratamentos/procedimentos (15). Em uma amostra de 101 descobertas da pesquisa experimental (aquela que é feita com animais ou células) que alegaram potencial terapêutico, somente cinco chegaram a ser testadas em humanos e somente uma chegou a ser utilizada amplamente na prática clínica duas décadas depois. Não é incomum estudos em animais serem em determinados contextos supervalorizados indevidamente (16).

Adicionalmente, a deturpação dos resultados pode implicar em outras consequências, como elevar expectativas falsas sobre tratamentos entre pacientes e clínicos (3), podendo também influenciar os responsáveis por formulações de políticas públicas a regular inadequadamente os tratamentos (por exemplo, a regulamentação sobre o efeito de uma determinada droga) ou até mesmo a incorporar políticas públicas equivocadas (17).

Spin em um artigo pode causar spin subsequente na notícia jornalística sobre o próprio artigo (caracterizando uma cascata de eventos, um efeito dominó), sendo bem comum artigos com spin serem propagados na imprensa $(8,11)$, essa que representa uma importante fonte de informação para público formado por não-cientistas, incluindo pacientes (18). No caso da calvície citado no início deste texto, somente adicionar a frase "em ratos" ao título da notícia poderia reduzir substancialmente as más interpretações, e a extrapolação gerada pela mesma poderia ser potencialmente evitada se o artigo original que deu fruto à notícia jornalística tivesse sido cético quanto aos seus próprios resultados (15), prevenindo o efeito dominó previamente mencionado $(9,12)$. Já se é documentado ser comum que artigos científicos e suas respetivas notícias jornalísticas demonstrem uma ênfase nos benefícios de descobertas científicas em detrimento dos malefícios ou da ausência de evidências sobre benefícios ou malefícios $(18,19)$.

Indivíduos sem ou com pouco treinamento científico ou estatístico em um determinado tema, ao lerem resumos de estudos em humanos e estudos em animais que contêm um determinado tratamento para uma determinada condição, afirmam com maior frequência que o tratamento é benéfico nos 
resumos dos estudos (o famoso abstract) que possuem spin, ou seja, que normalmente já forçam uma versão benéfica dos resultados independentemente da natureza dos resultados e dos dados brutos $(15,18)$. Tal fato ocorre entre os próprios cientistas, que também não estão isentos de interpretar erroneamente um estudo, portanto, não sendo necessariamente uma consequência de baixo letramento científico e estatístico (20). O resumo do artigo é lido mais frequentemente do que o texto completo (21) e por este motivo a presença de spin é bastante estudada em resumos de artigos (3).

Neste sentido, o spin alimenta uma narrativa heroica sobre o trabalho científico em que gênios isoladamente descobrem coisas e curam doenças (20). O problema desta narrativa é que ela não é verdadeira. $O$ trabalho científico, na verdade, é um esforço colaborativo e as descobertas tendem a ser discretas, incrementais e dependentes de contextos e condições muito particulares e específicas. Idealmente, os resultados da pesquisa científica devem ser relatados de forma completa, ética, transparente, reprodutível e acurada, sendo isso o que propõem as normas da ciência.

No entanto, as publicações científicas não são somente os dados brutos obtidos na pesquisa, também envolvendo escrita e argumentação. Neste processo, o spin pode ocorrer de forma até inconsciente pelos próprios cientistas no momento de escrever e argumentar os seus achados, podendo ser não intencional em sua natureza (20). Ele pode ocorrer de forma tão inconsciente que os estudos que quantificaram a presença de spin em outros estudos também foram passíveis de spin (22). O fato deste presente texto científico sobre spin apresentar uma possível fragilidade metodológica nos estudos científicos que estudam spin reflete o fato de que a ciência deve ser escrita de uma forma que contemple os dois (ou mais) lados/aspectos de uma investigação. Porque é assim que se faz ciência. Não se sabe de antemão o que pode ser encontrado. Supõe-se, testa-se, interpreta-se e relata-se da forma mais fidedigna e transparente possível.

No longo prazo, o spin pode contribuir para o emergente descrédito na ciência por parte da sociedade que tem que diariamente lidar com manchetes contraditórias (23). Por este motivo, o spin é um adoecimento de relato que deve ao máximo ser controlado (20). 
É importante ressaltar que, apesar do spin ser em grande parte estudado em estudos experimentais/intervenção ${ }^{1}$, os estudos observacionais ${ }^{2}$, que apresentam também grande apelo midiático (23) e, a depender dos casos um maior grau de flexibilidade de análises quantitativas e possíveis narrativas (24), também não ficam isentos de spin $(12,25,26)$. Tem-se como breve exemplo o artigo de Yang et al (2019) (26), publicado em uma das revistas científicas mais importantes da área médica (Journal of the American Medical Association JAMA), da área de educação física, uma área que apresenta amplo apelo midiático e interesse por parte da população (23).

O estudo observou indivíduos ao longo do tempo de forma retrospectiva ${ }^{3}$, a fim de estimar associações entre força muscular, medida pela capacidade de realizar flexões de braço, e eventos cardiovasculares (qualquer tipo de acometimento no sistema cardiovascular). Ao final do estudo, os autores encontraram associações negativas ${ }^{4}$ entre capacidade de realizar o exercício de flexão de braço e eventos cardiovasculares. No entanto, nas conclusões do resumo do artigo, visualiza-se uma alegação de um possível uso da flexão de braço como ferramenta para análise funcional. Tal alegação necessitaria de um estudo clínico e experimental para ser feita com segurança, caracterizando uma extrapolação do potencial do estudo, fato que por fim configura um tipo de spin também (25). A narrativa do estudo é tão tendenciosa que a flexão de braço é sugerida como uma medida simples, rápida e gratuita para avaliação clínica. Verificam-se inconsistências de narrativa entre o resumo do artigo e o texto completo, com o texto apresentando uma argumentação mais cética, baseada nos dados, e, portanto, menos tendenciosa $(25,26)$.

\footnotetext{
${ }^{1}$ Este é um modelo de estudo em que os indivíduos são alocados de forma aleatória para receber ou não um determinado procedimento que, presume-se, pode vir a ser benéfico.

2 É um tipo de estudo que observa as variáveis sem dar conta de ter grupos para controlá-las. Com isto, gera hipóteses para outros estudos mais robustos, mas não é capaz de evidenciar relações de causa e efeito de modo confirmatório.

${ }^{3}$ Modelo de estudo em que o pesquisador estuda os pacientes a partir de um desfecho/evento. Em outras palavras, ele deseja saber quais os fatores de risco influenciaram o surgimento do evento.

${ }^{4} \mathrm{~A}$ medida em que uma variável aumenta $(\mathrm{x})$, a outra variável diminui (y). No exemplo citado, a medida em que o número de flexão de braço decresce, o número de eventos cardiovasculares cresce/aumenta.
} 


\section{Dito isso, como identificar o spin no artigo científico?}

Basicamente, o spin pode ser concebido em 3 categorias, a saber: 1) relatos enganosos (relato enganoso de resultado é definido como relato incompleto dos resultados do estudo que podem ser enganosos para o leitor ${ }^{5}$; desfechos ${ }^{6}$ omitidos; etc.); 2) interpretação inadequada (interpretação inadequada dos resultados do estudo por parte dos próprios autores é definido como uma interpretação enganosa dos resultados do estudo que, em geral, superestimam os benefícios reais de uma intervenção) e 3) extrapolação inadequada dos resultados (é definido como uma extrapolação inapropriada dos resultados do estudo, especialmente quando recomendações clínicas são realizadas a partir desenhos observacionais, que não suportam tal condição ou extrapolações para populações não estudadas no artigo específico) (2).

Basicamente, cada categoria de spin inclui várias estratégias retóricas. Se são retóricas, não são baseadas em evidência, são baseadas em discurso. $\mathrm{Na}$ tabela 1, listaremos algumas das mais importantes para cada categoria e como o leitor poderá identificá-los.

\footnotetext{
${ }^{5}$ Por relato incompleto, entenda aquele que contém, por exemplo, uma estatística parcialmente apresentada.

${ }^{6}$ Desfecho é a principal medida do estudo. É a variável que depende da intervenção testada, observada ou mensurada. Por exemplo: se eu desejo verificar se exercício físico aumenta a força muscular, nesse caso, força muscular é o desfecho do meu estudo.
} 
Ausência de foco em danos Os resultados são reportados sem ou não reportar eventos avisos prévios sobre questões de adversos $^{7}$ segurança importantes
"Esse estudo mostrou que o tratamento $A$ foi melhor e mais tolerável que o tratamento B." No entanto, 30/100 participantes experimentaram eventos adversos decorrentes do tratamento e 5 interromperam o estudo.

"Nosso estudo demonstrou que os pacientes melhoraram a capacidade cardiorrespiratória e autoeficácia, quando foi comparado o modelo de exercício A e B". No entanto, dados que não apresentaram significância estatística ${ }^{8}$ não foram relatados (melhoria na qualidade do sono).
Somente um subconjunto de dados originais são reportados
Relato seletivo
Os avisos sobre eventos adversos encontram-

se, normalmente, na sessão de resultados do artigo ou em um item extra do estudo

chamado de material suplementar. Desconfie de estudos que não listem danos ou efeitos adversos ou que não informem que tentaram observá-los e mensurá-los. Muitas intervenções possuem consequências inesperadas e potencialmente danosas.

Normalmente, só há como saber se os autores reportaram apenas um conjunto de dados se você estiver em posse do protocolo de pesquisa ou do registro do ensaio clínico (estudo intervencionista em humanos) em alguma plataforma, para que se faça uma comparação. Exemplo: você observa o que foi escrito no protocolo de pesquisa (você pode ver se ele está disponível em alguma plataforma de acesso aberto - OSF ${ }^{9}$, por exemplo - ou pode solicitar aos autores) e compara com o que foi publicado. Então, tem como você verificar se houve (ou não) omissão de algum desfecho. Um outro caminho é verificar os objetivos que os autores anunciaram no resumo e o que a conclusão apresenta. Se o debate da conclusão não faz menção ao objetivo principal, é possível que os autores tenham optado por analisar um aspecto mais chamativo do problema pesquisado.

\footnotetext{
${ }^{7}$ Qualquer ocorrência médica indesejável em um paciente ou sujeito de investigação clínica que recebeu algum tipo de tratamento.

${ }^{8}$ Termo utilizado para verificar a discrepância de uma hipótese estatística em relação aos dados observados.

${ }^{9}$ Open Science Framework (OSF) é uma plataforma/repositório de acesso aberto.
} 
Descrição enganosa do

desenho do estudo

O desenho do estudo é apresentado

como robusto, mas na verdade, não é.

Qualquer palavra ou expressão enfatizando o efeito benéfico de uma determinada intervenção como adjetivos qualificando positivamente os achados e advérbios de intensidade

Não considerar as limitações do estudo Limitações importantes do estudo não resultados

Citar somente estudos que concordam com os resultados do presente estudo e, simultaneamente, ignorar estudos

Citação seletiva de outros estudos
"Com base nesse estudo podemos afirmar que leite causa malefícios em adultos. " Trata-se de uma revisão sem método sistemático de seleção - de inclusão ou exclusão - de artigos originais contendo relatos de pesquisa empírica, na qual os autores selecionam os artigos por conveniência, ou seja, há uma inclinação para selecionar apenas os trabalhos que mostram malefícios do leite ou que combinam melhor com a visão de mundo dos autores.
"O tratamento A foi bem tolerado, quando comparado com o tratamento $B$, e pode apresentar excelentes benefícios na qualidade de vida da população idosa".
Sugerimos que os leitores procurem um fluxograma contendo a estratégia de inclusão e de exclusão de artigos na revisão. Sem fluxograma, não há método, e sem método não há sistema. Se a revisão não é sistemática, ela não tem força para unificar e orientar a leitura da evidência científica disponível.

O leitor deve procurar por adjetivos e advérbios em lugar de relações claras e robustas de causalidade explicitada por comparações de grupos que receberam a intervenção em oposição a grupos que não receberam, dimensões que foram mensuradas numericamente em oposição a dimensões que não foram e assim por diante.

'No nosso estudo, vimos que a ingestão de suplementos antioxidantes pode prevenir doenças cardiovasculares". Não houve nenhum tipo de ajuste para variáveis que poderiam confundir - isto é, "melhorar" - o resultado (p. ex. exercício físico, alimentação, histórico familiar, etc.).

"Nosso estudo demonstrou que o exercício intervalado de alta intensidade promove maiores benefícios na capacidade cardiorrespiratória quando comparado com o aeróbico em intensidade

moderada". Vários estudos, incluindo meta-análises (estudos que sintetizam outros estudos sobre um tema), mostram que não há superioridade de método, principalmente quando o estudo é bem conduzido.
Em geral, todo estudo tem limitações e deve apresentar pelo menos um parágrafo sobre elas. Estudos que não apresentam esse parágrafo são estudos duvidosos.

Todo argumento científico preenche uma lacuna e toda lacuna é percebida numa contraditórios na intenção de sintetizá-los em um achado ou afirmação ou em uma negação ou em outra pergunta. Estudos cujos referenciais teóricos se apresentam muito homogêneos devem ser lidos com desconfiança em igual proporção. discussão robusta que contrapõe argumentos 


\section{Interpretação}

Reivindicar um determinado efeito para uma ausência de significância estatística
Uma determinada intervenção terapêutica é apresentada como efetiva apesar de os resultados não apresentarem significância estatística
A intervenção terapêutica e o

Reivindicar equivalência para comparador são apresentados como resultados não estatisticamente significativos, apesar de um amplo equivalentes quando um teste de comparação não é estatisticamente significante, e possui um grande uma incerteza grande para as intervalo de confiança, caracterizando extrapolações estatísticas e teóricas
"A dieta com estilo low-carb (baixo carboidrato) foi mais efetiva, quando comparada à dieta tradicional para redução da circunferência da cintura". No entanto, as estatísticas do artigo não indicam tal eficácia.
Normalmente, é posto que quando um valor de $\mathrm{p}$ (ou $p$-value) é menor que um ponto de corte de 0,05 não há significância estatística. De forma contrária, qualquer valor igual ou maior que 0,05 significa que há uma significância estatística. Em outras palavras, houve uma diferença numérica entre a média (ou outra medida) dos grupos, e ela foi estatisticamente significante.Nesse exemplo da dieta low-carb, o valor de P foi $>0,05$., então não houve diferença estatisticamente significativa. Em geral, esses dados são encontrados na sessão de resultados.

Os dados entre os dois grupos não apresentaram diferença significativa (nesse caso, houve um $p>0,05$ ), então os autores concluíram uma semelhança/equivalência entre os grupos. Mas, houve um amplo
S autores concluiram que: mortalidade dura "Ocompanhamento foi estatisticamente semelhante nos dois grupos (ou seja, presume-se sem confirmação estatística que o tratamento provavelmente não impactou na mortalidade)". intervalo de confianca (indicando incerteza entre os dados). O intervalo de confiança costuma aparecer em gráficos e tabelas na seção de resultados. Intervalos de confiança muito amplo são razão, em verdade, para DESconfiança.

\footnotetext{
${ }^{10}$ Tipo de estimativa por intervalo de um parâmetro populacional desconhecido. Usado para indicar a confiabilidade de uma estimativa.
} 
A intervenção terapêutica é

Excluindo a segurança de resultados sem significância estatística

upresentada como segura bas

significativo, apesar do amplo intervalo de confiança
"O tratamento prolongado com o fármaco $\mathrm{A}$ foi bem tolerado." No entanto, $17 \%$ dos participantes apresentaram eventos adversos decorrentes da intervenção.

Idealmente, os autores devem incluir, na sessão de resultados, dados sobre eventos adversos. No exemplo utilizado, os autores concluíram que o tratamento foi bem tolerado,

mas parte dos pacientes apresentou eventos adversos, os quais os autores preferiram não comentar em função da ausência de significância estatística. No entanto, possui um alto intervalo de confiança, indicando uma alta variabilidade entre os dados, indicando uma incerteza dos resultados. Novamente, fundamental atentar para o intervalo de confiança.

Nesse exemplo citado, sobretudo por ser um estudo transversal, não há como haver uma linguagem causal (causa e efeito) - obesidade causa hipertensão e diabetes tipo II - porque

"Em nosso estudo, observamos que obesidade causa hipertensão arterial e diabetes tipo II". Trat

de um estudo transversal ${ }^{11}$, onde não se pode
afirmar relação de causa e efeito (a causa precisa

Os resultados são apresentados como uma sentença que implica uma relação de causa-efeito entre a intervenção e os desfechos preceder ao efeito, necessariamente, e por isso a

temporalidade é necessária para estabelecimento de

causalidade).
.

se foi a obesidade ou a hipertensão $e$

diabetes. Os dados foram coletados em um único momento.

A linguagem causal está autorizada para relatar ensaios clínicos randomizados ${ }^{12}$, que manipulam uma ou mais variáveis para verificação da ocorrência (ou não) do desfecho. Então, dessa forma, você pode afirmar que se manipular variável $X$, variável $Y$ é modificada. Mesmo assim, as inferências causais devem ser feitas com cautela.

\footnotetext{
${ }^{11}$ Desenho de estudo que avalia os indivíduos em um recorte, não havendo temporalidade. Coleta todas as medidas em um único dia, normalmente.

12 Modelo de estudo experimental em que os indivíduos são alocados de forma aleatória para receber ou não um determinado procedimento preventivo, terapêutico ou paliativo. A unidade de análise é o indivíduo.
} 
Reivindicar somente diferenças significantes apesar da falta de teste estatístico

Título enganoso

Foco em resultados com significância estatística ao invés de levar em

consideração a significância clínica

\section{Extrapolação}

Extrapolação inadequada para uma larga população, intervenção ou desfechos
A intervenção e o comparador são comparados, apesar de não haver teste estatístico apropriado

O tratamento A é melhor do que o tratamento B para perda de peso. No entanto, quando o tratamento $A$ é combinado com tratamento $\mathrm{B}$, há uma melhora mais pronunciada no desfecho de interesse". Nenhum teste estatístico que fez essa comparação foi relatado.

"O uso do fármaco A melhora a síndrome metabólica

O título sugere resultados benéficos de uma determinada intervenção, mas não é suportado pelos resultados do estudo

"O adultos fisicamente inativos". Em realidade,

correu melhora de apenas 1 variável da síndrome metabólica. Os outros desfechos não apresentaram melhoras.

"A redução da circunferência do abdômen

Os resultados são apresentados através da significância estatística sem considerar a relevância clínica outras populações, intervenções

desfechos, além do que foi mostrado no bem como em doenças crônicas." A intervenção tev estudo (como resultados substitutos)
Nesse exemplo, o autor apenas fez uma

suposição/especulação de que quando junta o tratamento A com o B o desfecho é

melhorado. No entanto, para que essa

informação faça sentido, os autores precisam fazer testes estatísticos para avaliar se essa hipótese é verdadeira. Em geral, para tudo, quando se fala de hipóteses, é necessário que tenha um teste estatístico apropriado.

Normalmente, os autores tendem a utilizar

com cunho afirmativo, como o exemplo citado. O ideal é que o leitor veja na seção de resultados se, de fato, os dados apresentaram uma significância estatística para que os autores usassem um título com caráter afirmativo/verdadeiro.

Nessa sessão, infelizmente, não há algo palpável do que possui ou não significância clínica. Em geral, uma pequena

experiência/familiarização com a temática vai ajudar o leitor a saber o que possui ou não uma relevância clínica.

Nem sempre algo que é estatisticamente significativo é clinicamente relevante. Como regra, vale sempre ter em mente que afirmações extraordinárias requerem evidência extraordinária.

Em regra, nos resultados, discussão e conclusão devem constar apenas o que fo proposto em objetivos e métodos. Os esultados respondem a perguntas de investigação através de método. Nesse sentido, o que não foi perguntado não pode ser respondido. 
Nesse caso, não há um número certo para se classificar em "poucos pacientes". Mas não se deve fazer extrapolações quando se tem uma

Implicação inadequada para Os autores recomendam o uso de uma prática clínica

Os autores recomendam o uso de uma
determinada intervenção para prática clínica
'Não devemos hesitar a utilização do tratamento A

em desfecho X." Trata-se de um estudo prospectivo ${ }^{13}$ Idealmente, para todo estudo, é que se tenha com poucos pacientes.

um cálculo amostral ${ }^{14}$ para determinar

quantos pacientes serão avaliados e, dessa

forma, evite qualquer tipo de erro. Quando um

estudo não apresenta um cálculo amostral,

em geral, é motivo para desconfiança.

Tabela 1: Classificação dos tipos de spin em estudos de intervenção (adaptado e traduzido de Lazarus et al, 2015)

\footnotetext{
${ }^{13}$ Modelo de estudo em que o desfecho ainda não ocorreu, ou seja, os pacientes são acompanhados para ver a ocorrência do evento/desfecho.

${ }^{14}$ Um cálculo realizado para determinar o tamanho da amostra do estudo.
} 


\section{CONSIDERAÇÕES FINAIS}

E agora, devemos desistir da ciência, dos cientistas e do método científico? Não.

A ciência é um trabalho de descrença e ceticismo organizado (27) e é por isto que funciona: porque os próprios cientistas devem testar as suas crenças, pressupostos e hipóteses durante todo o tempo (28). Inclusive na redação do relato.

O conhecimento avança, através da ciência, porque ela não ser presume detentora do conhecimento, ela é somente uma guardiã do método e do rigor. Soa como um paradoxo? Não é. Estar aberto a novos conhecimentos é da própria natureza da construção do conhecimento.

Acreditamos que este trabalho irá servir como um guia para que os leitores (por exemplo, pacientes, jornalistas, ou até os próprios cientistas) possam ter uma leitura mais cética do conteúdo de muitos artigos científicos, principalmente os que possuem caráter duvidoso. É muito importante ressaltar que um artigo não é o relato de uma pesquisa pronta, é o estágio inicial da vida pública da pesquisa. Ele foi publicado para ser lido criticamente, debatido, contestado, confirmado, problematizado.

Provavelmente, spin em textos científicos e não científicos (comunicados de imprensa, por exemplo) não acabarão. Então, para cientistas e não cientistas, deixamos as seguintes recomendações:

a) Identifique se a afirmação dita tem base científica que a suporte no texto;

b) Identifique se há a presença de termos sensacionalistas como "cura", "milagre", "sensacional" e adjetivações que não sejam apoiadas em quantificações;

c) Identifique se o estudo foi realizado em humanos ou em animais/células;

d) Faça uso de sua análise crítica para descartar possíveis confundidores na estrutura lógica da argumentação e do método;

e) Não leia somente o título e resumo dos artigos. Eles costumam ser redutivos e não passar a informação completa;

f) Não considere um artigo verdadeiro somente porque ele está publicado numa revista comercial famosa. Revistas famosas também erram;

g) Não tome decisões com base em apenas um artigo. Avalie, se possível, o corpo completo de evidências. 
Por fim, nós acreditamos que a apresentação dos dados precisa ser íntegra e transparente independentemente do estudo, tanto em no resumo como em textos completos. Isso facilitará uma leitura mais cética e menos enganosa para potenciais leitores da pesquisa biomédica e ajudará a ciência a cumprir o seu papel social de melhorar a vida de todos um passo de cada vez.

\section{REFERÊNCIAS}

1. Boutron I, Dutton S, Ravaud P, Altman DG. Reporting and interpretation of randomized controlled trials with statistically nonsignificant results for primary outcomes. JAMA. 2010;303(20):2058-64.

2. Lazarus C, Haneef R, Ravaud P, Boutron I. Classification and prevalence of spin in abstracts of non-randomized studies evaluating an intervention. BMC Med Res Methodol. 2015;15:85.

3. Boutron I, Altman DG, Hopewell S, Vera-Badillo F, Tannock I, Ravaud P. Impact of spin in the abstracts of articles reporting results of randomized controlled trials in the field of cancer: the SPIIN randomized controlled trial. J Clin Oncol. 2014;32(36):41206.

4. Horton R. The rhetoric of research. BMJ. 1995;310(6985):985-7.

5. Kalaichandran A. Worried About That New Medical Study? Read This First. New York Times 2020. Available from: https://parenting.nytimes.com/health/medical-studyconcerns?module=ptg-onsite-share\&type=email.

6. Haneef R, Lazarus C, Ravaud P, Yavchitz A, Boutron I. Interpretation of Results of Studies Evaluating an Intervention Highlighted in Google Health News: A CrossSectional Study of News. PLoS One. 2015;10(10):e0140889.

7. Ochodo EA, de Haan MC, Reitsma JB, Hooft L, Bossuyt PM, Leeflang MM. Overinterpretation and misreporting of diagnostic accuracy studies: evidence of "spin". Radiology. 2013;267(2):581-8.

8. Yavchitz A, Boutron I, Bafeta A, Marroun I, Charles P, Mantz J, et al. Misrepresentation of randomized controlled trials in press releases and news coverage: a cohort study. PLoS Med. 2012;9(9):e1001308.

9. Woloshin S, Schwartz LM, Casella SL, Kennedy AT, Larson RJ. Press releases by academic medical centers: not so academic? Ann Intern Med. 2009;150(9):613-8.

10. Cummings P, Rivara FP. Spin and boasting in research articles. Arch Pediatr Adolesc Med. 2012;166(12):1099-100.

11. Sumner P, Vivian-Griffiths S, Boivin J, Williams A, Venetis CA, Davies A, et al. The association between exaggeration in health related science news and academic press releases: retrospective observational study. BMJ. 2014;349:g7015.

12. Cofield SS, Corona RV, Allison DB. Use of causal language in observational studies of obesity and nutrition. Obes Facts. 2010;3(6):353-6.

13. Moynihan R, Bero L, Ross-Degnan D, Henry D, Lee K, Watkins J, et al. Coverage by the news media of the benefits and risks of medications. N Engl J Med. 2000;342(22):1645-50.

14. Brown AW, Bohan Brown MM, Allison DB. Belief beyond the evidence: using the proposed effect of breakfast on obesity to show 2 practices that distort scientific evidence. Am J Clin Nutr. 2013;98(5):1298-308. 
15. Crowe K. Mice are not people: Fighting spin in medical science CBC2019. Available from: https://www.cbc.ca/news/health/mice-science-research-spin-newsmedia-1.5194985.

16. Contopoulos-loannidis DG, Alexiou GA, Gouvias TC, Ioannidis JP. Medicine. Life cycle of translational research for medical interventions. Science. 2008;321(5894):1298-9.

17. BMJ. Tamiflu Campaign The BMJ. Available from: https://www.bmj.com/tamiflu. 18. Boutron I, Haneef R, Yavchitz A, Baron G, Novack J, Oransky I, et al. Three randomized controlled trials evaluating the impact of "spin" in health news stories reporting studies of pharmacologic treatments on patients/'caregivers' interpretation of treatment benefit. BMC Med. 2019;17(1):105.

19. Bubela TM, Caulfield TA. Do the print media "hype" genetic research? A comparison of newspaper stories and peer-reviewed research papers. CMAJ. 2004;170(9):1399-407.

20. Boutron I, Ravaud P. Misrepresentation and distortion of research in biomedical literature. Proc Natl Acad Sci USA. 2018;115(11):2613-9.

21. Altwairgi AK, Booth CM, Hopman WM, Baetz TD. Discordance between conclusions stated in the abstract and conclusions in the article: analysis of published randomized controlled trials of systemic therapy in lung cancer. J Clin Oncol. 2012;30(28):3552-7.

22. Bero L, Chiu K, Grundy Q. The SSSPIN study-spin in studies of spin: metaresearch analysis. BMJ. 2019;367:I6202.

23. Ioannidis JPA. Neglecting Major Health Problems and Broadcasting Minor, Uncertain Issues in Lifestyle Science. JAMA. 2019:1-2.

24. Patel CJ, Burford B, loannidis JP. Assessment of vibration of effects due to model specification can demonstrate the instability of observational associations. J Clin Epidemiol. 2015;68(9):1046-58.

25. Prasad V, Jorgenson J, loannidis JP, Cifu A. Observational studies often make clinical practice recommendations: an empirical evaluation of authors' attitudes. J Clin Epidemiol. 2013;66(4):361-6.e4.

26. Yang J, Christophi CA, Farioli A, Baur DM, Moffatt S, Zollinger TW, et al. Association Between Push-up Exercise Capacity and Future Cardiovascular Events Among Active Adult Men. JAMA Netw Open. 2019;2(2):e188341.

27. Merton RK. Ensaios de sociologia da ciência. Brasil: Editora 34; 2013.

28. Ziman J. O conhecimento confiável: uma exploração dos fundamentos para a crença na ciência. Campinas: Papirus; 1996. 